\title{
Drug Mechanisms to Help in Managing Resistant Hypertension in Obesity
}

\author{
Pieter M. Jansen - Jan A. H. Danser • Wilko Spiering • \\ Anton H. van den Meiracker
}

Published online: 8 June 2010

(C) The Author(s) 2010. This article is published with open access at Springerlink.com

\begin{abstract}
Obesity is a major risk factor for the development of hypertension. Because the prevalence of obesity is increasing worldwide, the prevalence of obesity hypertension is also increasing. Importantly, hypertension in obesity is commonly complicated by dyslipidemia and type 2 diabetes mellitus and hence imposes a high cardiovascular disease risk. Furthermore, obesity is strongly associated with resistant hypertension. Activation of the sympathetic nervous system and the renin-angiotensin system, leading to renal sodium and water retention, links obesity with hypertension. There is also evidence for the release of factors by visceral adipose tissue promoting excessive aldosterone production, and a more central role of aldosterone in obesity hypertension is emerging. Randomized studies evaluating the effect of different classes of antihypertensive agents in obesity hypertension are scarce, short-lasting, and small. Considering the emerging role of aldosterone in the pathogenesis of obesity hypertension, mineralocorticoid receptor antagonism may play a more central role in the pharmacologic treatment of obesity hypertension in the near future.
\end{abstract}

Keywords Obesity · Hypertension · Aldosterone · Sympathetic nervous system · Renin-angiotensin system . Antihypertensive therapy $\cdot$ Mineralocorticoid receptor antagonism

P. M. Jansen · J. A. H. Danser · A. H. van den Meiracker $(\square)$ Division of Pharmacology, Vascular and Metabolic Diseases, Department of Internal Medicine, Erasmus MC,

Room D432, 's Gravendijkwal 230,

3015CE Rotterdam, The Netherlands

e-mail: a.vandenmeiracker@erasmusmc.nl

W. Spiering

Department of Vascular Medicine, UMC Utrecht,

Room F02. 126,

3508 GA Utrecht, The Netherlands

\section{Introduction}

Obesity, and especially central obesity, is one of the most common risk factors for the development of hypertension. Population studies indicate that two thirds of the prevalence of hypertension can be attributed to overweight or obesity [1]. In the National Health and Examination Survey (NHANES) III, the prevalence of hypertension according to body mass index (BMI) and adjusted for age was about $14 \%$ in participants with a BMI less than $25 \mathrm{~kg} / \mathrm{m}^{2}$ versus about $40 \%$ in participants with a BMI greater than $30 \mathrm{~kg} / \mathrm{m}^{2}$ [2]. Obesity hypertension is commonly accompanied by type 2 diabetes mellitus and dyslipidemia [3]. Furthermore, obesity hypertension is associated with increased risks for left ventricular hypertrophy, microalbuminuria, and renal hyperfiltration. Over time, the latter two factors predispose to impairment of renal function that may further aggravate hypertension [3].

Obesity is also the most important risk factor for the syndrome of obstructive sleep apnea (OSA); apart from obesity itself, OSA also can contribute to the development and maintenance of hypertension [4]. Finally, obesity and OSA are well-recognized patient characteristics that, along with older age, a higher baseline systolic blood pressure, and left ventricular hypertrophy, are associated with treatment-resistant hypertension $[5,6]$.

\section{Obesity Hypertension: The Underlying Mechanism}

Obesity hypertension is a complex condition and, as is true for hypertension in general, impaired pressure natriuresis is a key etiologic factor [7] (Fig. 1). The renal sodium retention in obesity hypertension occurs despite an increase in glomerular filtration rate, indicating increased renal 
Fig. 1 Mechanisms linking obesity with hypertension

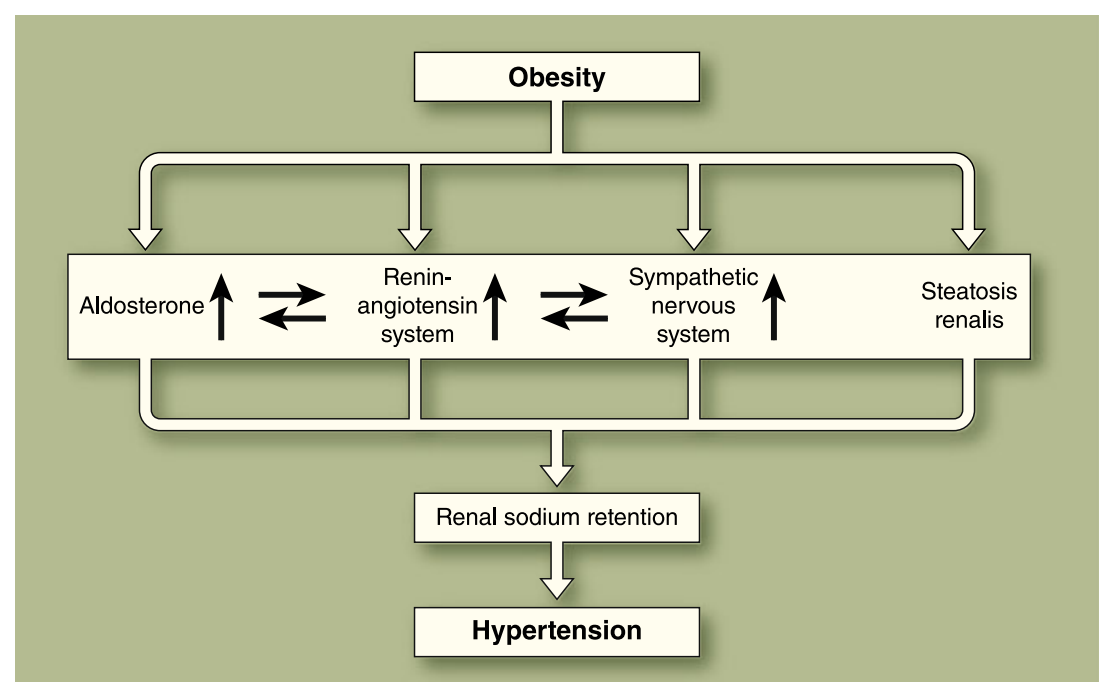

fractional tubular sodium reabsorption [8]. Driving forces for this sodium retention are an increase in renal sympathetic tone and activation of the renin-angiotensin system (RAS). Moreover, renal medullary compression by excessive adipose tissue, for which we propose the term steatosis renalis (analogous to steatosis hepatis), may also account for avid sodium retention [8]. Although renal plasma flow and glomerular filtration rate are increased in the initial phase of obesity hypertension to cope with the increased metabolic demands, glomerular injury contributing to the maintenance of hypertension is a feared complication in the long run [9].

Evidence that these mechanisms are involved in obesity hypertension comes mainly from experimental studies. Thus the development of obesity-induced hypertension in dogs exposed to a high-fat diet could be prevented by renal denervation and by agents that interfere with the renin-angiotensin system $[10,11]$. Moreover, in a small clinical study, combined alphaadrenergic and beta-adrenergic receptor blockade induced a greater reduction in blood pressure in obese than in lean hypertensive patients [12].

\section{Obesity and Resistant Hypertension: The Emerging Role of Aldosterone}

Resistant hypertension is defined as blood pressure that remains above the target value in spite of the concurrent use of three antihypertensive agents of different classes. Ideally, one of the three classes should be a diuretic and all agents should be prescribed at optimal doses [13]. The prevalence of resistant hypertension is unknown. In an analysis of NHANES III, only $53 \%$ of the population being treated for hypertension were controlled to a blood pressure less than 140/90 mm Hg [14]. Blood pressure remains uncontrolled most often because of persistent elevation of systolic blood pressure. Patient characteristics associated with resistant hypertension are older age, obesity, the presence of left ventricular hypertrophy, and chronic kidney disease $[5,6$, 15]. In a large cross-sectional study of 45,125 primary care patients, those with a BMI of $40 \mathrm{~kg} / \mathrm{m}^{2}$ or higher not only had a higher prevalence of hypertension, but also had a 5.3-fold higher probability of requiring four antihypertensive agents to achieve blood pressure control, compared with normal-weight patients, and they had a 3.2-fold probability of requiring three agents [16]. Studies performed in patients with resistant hypertension showed that the prevalence of primary aldosteronism (PA) is $17 \%$ to $22 \%[17,18]$. Patients with resistant hypertension without PA had higher plasma aldosterone levels and urinary aldosterone excretion rates and a larger intravascular volume than normotensive individuals [19]. The role of aldosterone in the metabolic syndrome has emerged more recently. In the Framingham offspring study, higher aldosterone levels were predictive for the development of the metabolic syndrome [20]. In blacks, plasma aldosterone, but not plasma renin activity, was higher in individuals with the metabolic syndrome than in those without, and a significant association between waist circumference and plasma aldosterone concentration could be established [21]. Moreover, weight reduction in obese individuals results in a decrease in plasma aldosterone concentration, providing further evidence for a stimulating effect of adipose tissue on adrenal aldosterone production $[22,23]$. Although several mechanisms may underlie these higher aldosterone concentrations, one possibility is that it relates to the secretion of a mineralocorticoid-releasing factor by adipocytes $[24,25 \bullet \cdot$. This idea is of interest, given the concept of angiotensinogen release from adipose tissue and the documented synergistic action between aldosterone and angiotensin II [26-28]. 
The beneficial effect of low-dose spironolactone added to combinations of first-line antihypertensive agents in patients with resistant hypertension has been shown in several studies $[29,30 \bullet, 31-34]$. As shown in Table 1, almost all patients included in these studies were overweight (BMI, $25-30 \mathrm{~kg} / \mathrm{m}^{2}$ ) or obese (BMI $>30 \mathrm{~kg} / \mathrm{m}^{2}$ ). Based on the findings of these studies and the knowledge that plasma aldosterone is increased in obesity, aldosterone receptor antagonism seems a logical therapeutic option in obesity hypertension.

\section{Randomized Trials with Antihypertensive Agents in Obesity Hypertension}

Although obesity-associated hypertension is a highly prevalent condition, randomized studies evaluating the effect of specific classes of antihypertensive agents in this condition are scarce, small, and of short duration. Most of these studies investigated not only blood pressure reduction but also the occurrence of potential adverse metabolic effects. The Treatment in Obese Patients With Hypertension (TROPHY) trial is the first randomized, double-blind, placebo-controlled trial comparing the effects of the angiotensin-converting enzyme (ACE) inhibitor lisinopril (maximal dose, $40 \mathrm{mg}$ once daily) with hydrochlorothiazide (HCT, maximal dose $50 \mathrm{mg}$ once daily) in obesity hypertension [35]. In total, 232 patients (mean age, 52 years) with a mean BMI of $32 \mathrm{~kg} / \mathrm{m}^{2}$ were enrolled. Study medication was given for 12 weeks. At the end of the study, office and ambulatory blood pressures were reduced to a similar extent with both regimens, but the response rate was not higher than $40 \%$ in the lisinopril group and $33 \%$ in the HCT group, in accordance with the knowledge that a combination of different antihypertensive agents is almost always required for blood pressure control. Although neither treatment significantly affected serum insulin or lipid concentrations, glucose increased modestly with HCT compared with lisinopril (difference, $0.5 \mathrm{mmol} / \mathrm{L}$ ). On the basis of these findings, the authors concluded that a rational monotherapy approach for initial treatment of obese patients with hypertension may include an ACE inhibitor or a diuretic.

The CROSS study (Candesartan Role on Obesity and on Sympathetic System) was published in 2003 [36]. In this study, which enrolled 127 patients with an average BMI of $33 \mathrm{~kg} / \mathrm{m}^{2}$, the effect of candesartan (8-16 mg daily) was compared with HCT (25-50 mg daily) using a parallelgroup, double-blind study design. Study medication was given for 12 weeks. Blood pressure reduction with both regimens was identical, with blood pressure normalization in about $80 \%$ of patients. Compared with HCT, candesartan was associated with improved insulin sensitivity and modestly lower muscle sympathetic nerve activity. (It should be remarked that these two measurements were performed in only a small fraction of the patients.)

In a third study the effects of valsartan, $160 \mathrm{mg}$ daily, were compared with those of atenolol, $100 \mathrm{mg}$ daily, in a double-blind, parallel-group study that included 132 patients with an average BMI of $33 \mathrm{~kg} / \mathrm{m}^{2}$ and a waist circumference of $110 \mathrm{~cm}$ [37]. Study medication was given for 12 weeks. If blood pressure was not normalized after 4 weeks monotherapy with valsartan or atenolol, HCT (12.5-25 mg daily) could be added. An interesting and unanticipated finding of this study was that the diastolic blood pressure reduction was greater with monotherapy atenolol than with valsartan. HCT was added to $49 \%$ of the patients allocated to atenolol, compared with $70 \%$ of the patients allocated to valsartan. At the end of the treatment period, blood pressure reduction with the two regimens was identical. No difference in serum lipids between the two regimens was observed, whereas in the atenolol group the homeostasis model assessment (HOMA) insulin index slightly worsened in insulin-sensitive patients but not in insulin-resistant patients. The authors concluded that betaadrenoceptor blockers and angiotensin II type $1\left(\mathrm{AT}_{1}\right)$ receptor blockers, particularly in combination with low-dose

Table 1 Summary of studies evaluating the effect of add-on spironolactone treatment in uncontrolled or resistant hypertension

\begin{tabular}{|c|c|c|c|c|c|c|c|c|}
\hline Study & Design & $N$ & $\begin{array}{l}\mathrm{BW}, \\
\mathrm{kg}\end{array}$ & $\begin{array}{l}\mathrm{BMI}, \\
\mathrm{kg} / \mathrm{m}^{2}\end{array}$ & $\begin{array}{l}\text { Waist } \\
\text { circumference, } \\
\mathrm{cm}\end{array}$ & $\begin{array}{l}\text { Antihypertensives, } \\
n\end{array}$ & $\begin{array}{l}\text { Spironolactone } \\
\text { dose, } m g / \text { day }\end{array}$ & $\begin{array}{l}\text { BP reduction, } \\
\mathrm{mm} \mathrm{Hg}\end{array}$ \\
\hline Ouzan et al. [32] & Uncontrolled & 25 & NA & NA & NA & 3.2 & $1 \mathrm{mg} / \mathrm{kg}$ & $24 / 10$ \\
\hline \multirow[t]{2}{*}{ Saha et al. [33] } & \multirow[t]{2}{*}{ Placebo-controlled } & 23 (spironolactone) & NA & 32.0 & NA & 2 & 25 & $5 / 2$ \\
\hline & & 27 (placebo) & NA & 35.7 & NA & 2 & - & - \\
\hline Sharabi et al. [34] & Uncontrolled & 42 & NA & 30.6 & NA & $\geq 2$ & $12.5-25$ & $23.2 / 12.5$ \\
\hline Lane et al. [31] & Uncontrolled & 119 & 86.7 & NA & NA & 3.7 & $25-100$ & $21.7 / 8.5$ \\
\hline Chapman et al. [29] & Uncontrolled & 1411 & & 29.4 & NA & $\geq 3$ & $25-50$ & $21.9 / 9.5$ \\
\hline de Souza et al. $[30 \bullet]$ & Uncontrolled & 175 & & 30.2 & 100 & $\geq 3$ & $25-100$ & $14 / 7$ \\
\hline
\end{tabular}

$B M I$ body mass index, $B W$ body weight, $N A$ not available 
diuretics, effectively lower blood pressure in obese patients, but that $\mathrm{AT}_{1}$-receptor blockers may be preferred because of the high diabetes risk in obesity.

In a more recent study, the direct renin inhibitor aliskiren has been compared with irbesartan and amlodipine in a placebocontrolled trial in obese hypertensive patients in whom blood pressure was still elevated after HCT monotherapy for 4 weeks [38]. A total of 489 patients with an average BMI of about $34 \mathrm{~kg} / \mathrm{m}^{2}$ were included. The mean reduction in blood pressure in the three active groups was identical. Rates of blood pressure control were $34 \%$ in the HCT group, $57 \%$ in the aliskiren/HCT group, 55\% in the irbesartan/HCT group, and $45 \%$ in the amlodipine/HCT group. Adverse effects, mainly due to a higher incidence of peripheral edema, were highest in the amlodipine/HCT group.

Although these studies were not performed in patients with resistant hypertension, they all demonstrate that obesity hypertension responds to a variety of antihypertensive agents. As known from many studies in high-risk populations, the incidence of new-onset diabetes mellitus is increased with the use of beta-blockers and thiazide diuretics, compared with other antihypertensive agents [39]. Recently, it was shown that adverse metabolic effects, including new-onset diabetes, already occurred after short-term (9-18 weeks) exposure to atenolol, HCT, or their combination and that this effect was more common in patients with abdominal obesity [40]. Because the treatment of hypertension usually requires lifelong therapy and the likelihood of developing adverse metabolic effects increases with increasing exposure duration, not only the blood pressure lowering properties but also the anticipated adverse metabolic effects should be considered when these agents are prescribed.

\section{Mineralocorticoid Receptor Antagonism in Obesity Hypertension}

In our view, the accumulating evidence of the role of aldosterone excess in the pathogenesis of obesity hypertension and the knowledge that mineralocorticoid receptor blockers promote additional blood pressure reduction in patients with resistant or difficult-to-control hypertension provide a firm basis for a more liberal use of spironolactone (or its more selective counterpart, eplerenone) in obesity hypertension. In this regard it should be noted that, in the reported studies on the additional effect of spironolactone in uncontrolled or resistant hypertension, BMI was $30 \mathrm{~kg} / \mathrm{m}^{2}$ or higher in most of the populations studied (Table 1). Furthermore, a recent prospective study performed in patients with true resistant hypertension who had an average BMI of $30 \mathrm{~kg} / \mathrm{m}^{2}$ and waist circumference of $100 \mathrm{~cm}$ showed in a multivariate regression analysis that a higher waist circumference and an increased pulse wave velocity were the two independent variables that predicted the systolic blood pressure response to spironolactone [30 0 .

\section{Lifestyle Modification and Interaction with Antihypertensive Regimens}

Without doubt, weight loss and reduction of dietary sodium intake, although not specifically evaluated in patients with resistant obesity hypertension, have a clear benefit in reducing blood pressure and often allow a reduction in the number of prescribed medications $[41,42 \cdot \bullet$ ]. A meta-analysis of longterm weight loss studies indicates that a $10-\mathrm{kg}$ weight loss is associated with a blood pressure reduction of $6.0 \mathrm{~mm} \mathrm{Hg}$ systolic and $4.6 \mathrm{~mm} \mathrm{Hg}$ diastolic [43]. Therefore, weight reduction and maintenance of weight loss are important steps in managing obesity hypertension. Unfortunately, nonpharmacologic approaches aimed at reducing body weight have limited sustainability in most patients, and adjuvant pharmacotherapy may be necessary for effective weight reduction and its maintenance [44].

The effectiveness of drug-based weight loss with sibutramine on the basis of different antihypertensive regimens was addressed in the Hypertension-Obesity-Sibutramine (HOS) study [45]. In this prospective, double-blind, placebocontrolled study, 171 patients receiving one of three antihypertensive combination therapies (felodipine plus ramipril, verapamil plus trandolapril, or metoprolol plus HCT) were assigned randomly to sibutramine ( $15 \mathrm{mg}$ daily) or placebo for a period of 16 weeks. Compared with placebo, sibutramine resulted in a significantly greater decrease in body weight (5.7 vs $1.5 \mathrm{~kg})$, BMI (2.0 vs $\left.0.5 \mathrm{~kg} / \mathrm{m}^{2}\right)$ and waist circumference $(5.0$ vs $0.8 \mathrm{~cm})$. Remarkably, the sibutramine-induced weight loss, reduction in BMI, and reduction in waist circumference were significantly attenuated in the metoprolol plus HCT group compared with the other two groups. Despite the greater decrease in body weight, changes in office systolic and diastolic blood pressure did not differ between the sibutramine and placebo group. Ambulatory blood pressure recordings for $24 \mathrm{~h}$ revealed a small reduction in systolic blood pressure in both groups $(0.3 \mathrm{~mm} \mathrm{Hg}$ with sibutramine vs $0.9 \mathrm{~mm} \mathrm{Hg}$ in the placebo group), but unfortunately an increase in diastolic blood pressure in the sibutramine group versus placebo (2.1 versus $-0.3 \mathrm{~mm} \mathrm{Hg}$ ). The smaller decrease in body weight, BMI, and waist circumference with the use of sibutramine in the patients treated with metoprolol plus HCT was accompanied by less beneficial effects on glucose tolerance and hypertriglyceridemia. On the basis of these findings, the authors concluded that an antihypertensive combination therapy regimen that is based on ACE inhibitors and calcium channel blockers is more advantageous than a combination regimen based on diuretics and beta-blockers in supporting the weight-reduction 
actions and concomitant metabolic changes induced by sibutramine in obese hypertensive subjects.

\section{Conclusions}

Obesity hypertension is a growing condition. In a proportion of patients with obesity hypertension, the response of blood pressure to combinations of antihypertensive agents is insufficient. For these patients, no specific recommendations or guidelines regarding additional treatment are available. In light of the emerging role of aldosterone in the pathogenesis of obesity hypertension and the findings of studies suggesting that adding a low dose of aldosterone can produce large blood pressure reductions in patients whose blood pressure is uncontrolled with conventional antihypertensive treatment, addition of a mineralocorticoid receptor antagonist as a fourthline agent in resistant obesity hypertension is a logical choice. Conventional or nonvasodilating beta-blockers, although effective in reducing blood pressure in obesity hypertension, are not recommended as a first-line, second-line, or third-line agent because they reduce energy expenditure, lipolysis, and insulin sensitivity, and a negative interaction with sibutramine has been documented when they are combined with thiazide diuretics $[45,46]$. The chance of developing diabetes mellitus is greater with thiazide diuretics than with agents that interfere with the RAS or with calcium channel blockers $[47,48]$. This negative effect, among others, has been linked with the reduction in potassium and magnesium levels [49]. This effect may be less apparent when thiazide diuretics are combined with anti-RAS agents such as ACE inhibitors or $\mathrm{AT}_{1}$-receptor blockers or with mineralocorticoid receptor antagonists.

\section{Acknowledgment PMJ is supported by the Dutch Kidney Foundation,} grant C05-2151.

Disclosure No potential conflicts of interest relevant to this article were reported.

Open Access This article is distributed under the terms of the Creative Commons Attribution Noncommercial License which permits any noncommercial use, distribution, and reproduction in any medium, provided the original author(s) and source are credited.

\section{References}

Recently published papers of interest have been highlighted as:

- Of importance

•• Of major importance

1. Jordan J, Engeli S, Redon J, et al.: European Society of Hypertension Working Group on Obesity: background, aims and perspectives. J Hypertens 2007, 25(4):897-900.
2. Brown CD, Higgins M, Donato KA, et al.: Body mass index and the prevalence of hypertension and dyslipidemia. Obes Res 2000, 8(9):605-619.

3. Sharma AM: Is there a rationale for angiotensin blockade in the management of obesity hypertension? Hypertension 2004, 44 (1):12-19.

4. Peppard PE, Young T, Palta M, Skatrud J: Prospective study of the association between sleep-disordered breathing and hypertension. N Engl J Med 2000, 342(19):1378-1384.

5. Cushman WC, Ford CE, Cutler JA, et al.: Success and predictors of blood pressure control in diverse North American settings: the antihypertensive and lipid-lowering treatment to prevent heart attack trial (ALLHAT). J Clin Hypertens (Greenwich) 2002, 4 (6):393-404.

6. Lloyd-Jones DM, Evans JC, Larson MG, et al.: Differential control of systolic and diastolic blood pressure: factors associated with lack of blood pressure control in the community. Hypertension 2000, 36 (4):594-599.

7. Davy KP, Hall JE: Obesity and hypertension: two epidemics or one? Am J Physiol Regul Integr Comp Physiol 2004, 286(5): R803-R813.

8. Hall JE: The kidney, hypertension, and obesity. Hypertension 2003, 41(3 Pt 2):625-633.

9. Bagby SP: Obesity-initiated metabolic syndrome and the kidney: a recipe for chronic kidney disease? J Am Soc Nephrol 2004, 15 (11):2775-2791.

10. de Paula RB, da Silva AA, Hall JE: Aldosterone antagonism attenuates obesity-induced hypertension and glomerular hyperfiltration. Hypertension 2004, 43(1):41-47.

11. Kassab S, Kato T, Wilkins FC, et al.: Renal denervation attenuates the sodium retention and hypertension associated with obesity. Hypertension 1995, 25(4 Pt 2):893-897.

12. Wofford MR, Anderson DC Jr, Brown CA, et al.: Antihypertensive effect of alpha- and beta-adrenergic blockade in obese and lean hypertensive subjects. Am J Hypertens 2001, 14(7 Pt 1):694-698.

13. Calhoun DA, Jones D, Textor S, et al.: Resistant hypertension: diagnosis, evaluation, and treatment. A scientific statement from the American Heart Association Professional Education Committee of the Council for High Blood Pressure Research. Hypertension 2008, 51(6):1403-1419.

14. Hajjar I, Kotchen TA: Trends in prevalence, awareness, treatment, and control of hypertension in the United States, 1988-2000. JAMA 2003, 290(2):199-206.

15. Nishizaka MK, Pratt-Ubunama M, Zaman MA, et al.: Validity of plasma aldosterone-to-renin activity ratio in African American and white subjects with resistant hypertension. Am J Hypertens 2005, 18(6):805-812.

16. Bramlage P, Pittrow D, Wittchen HU, et al.: Hypertension in overweight and obese primary care patients is highly prevalent and poorly controlled. Am J Hypertens 2004, 17(10):904-910.

17. Jansen PM, Boomsma F, van den Meiracker AH, Dutch AI: Aldosterone-to-renin ratio as a screening test for primary aldosteronism - the Dutch ARRAT Study. Neth J Med 2008, 66 (5):220-228.

18. Jansen PM, Danser AH, Imholz BP, van den Meiracker AH: Aldosterone-receptor antagonism in hypertension. J Hypertens 2009, 27(4):680-691.

19. Gaddam KK, Nishizaka MK, Pratt-Ubunama MN, et al.: Characterization of resistant hypertension: association between resistant hypertension, aldosterone, and persistent intravascular volume expansion. Arch Intern Med 2008, 168(11):1159-1164.

20. Ingelsson E, Pencina MJ, Tofler GH, et al.: Multimarker approach to evaluate the incidence of the metabolic syndrome and longitudinal changes in metabolic risk factors: the Framingham Offspring Study. Circulation 2007, 116(9):984-992. 
21. Kidambi S, Kotchen JM, Grim CE, et al.: Association of adrenal steroids with hypertension and the metabolic syndrome in blacks. Hypertension 2007, 49(3):704-711

22. Dall'Asta C, Vedani P, Manunta P, et al.: Effect of weight loss through laparoscopic gastric banding on blood pressure, plasma renin activity and aldosterone levels in morbid obesity. Nutr Metab Cardiovasc Dis 2009, 19(2):110-114.

23. Tuck ML, Sowers J, Dornfeld L, et al.: The effect of weight reduction on blood pressure, plasma renin activity, and plasma aldosterone levels in obese patients. N Engl J Med 1981, 304(16):930-933.

24. Goodfriend TL, Calhoun DA: Resistant hypertension, obesity, sleep apnea, and aldosterone: theory and therapy. Hypertension 2004, 43(3):518-524.

25. •- Sowers JR, Whaley-Connell A, Epstein M: Narrative review: the emerging clinical implications of the role of aldosterone in the metabolic syndrome and resistant hypertension. Ann Intern Med 2009, 150(11):776-783. This is an excellent review about underlying mechanisms and potential links between aldosterone, insulin resistance, and hypertension.

26. Chai W, Garrelds IM, de Vries R, et al.: Nongenomic effects of aldosterone in the human heart: interaction with angiotensin II. Hypertension 2005, 46(4):701-706.

27. Frederich RC Jr, Kahn BB, Peach MJ, Flier JS: Tissue-specific nutritional regulation of angiotensinogen in adipose tissue. Hypertension 1992, 19(4):339-344.

28. Massiera F, Bloch-Faure M, Ceiler D, et al.: Adipose angiotensinogen is involved in adipose tissue growth and blood pressure regulation. FASEB J 2001, 15(14):2727-2729.

29. Chapman N, Dobson J, Wilson S, et al.: Anglo-Scandinavian Cardiac Outcomes Trial I: effect of spironolactone on blood pressure in subjects with resistant hypertension. Hypertension 2007, 49(4):839-845.

30. - de Souza F, Muxfeldt E, Fiszman R, Salles G: Efficacy of spironolactone therapy in patients with true resistant hypertension. Hypertension 2010, 55(1):147-152. This study explores the effect and determinants of antihypertensive response in patients with true resistant hypertension. In this study, blood pressure was reduced by 16 and $9 \mathrm{~mm} \mathrm{Hg}$ with add-on spironolactone therapy, and controlled ambulatory blood pressure was reached in $48 \%$ of patients. Independent factors associated with better response were higher waist circumference, lower aortic pulse wave velocity, and lower serum potassium concentration.

31. Lane DA, Shah S, Beevers DG: Low-dose spironolactone in the management of resistant hypertension: a surveillance study. J Hypertens 2007, 25(4):891-894.

32. Ouzan J, Perault C, Lincoff AM, et al.: The role of spironolactone in the treatment of patients with refractory hypertension. Am J Hypertens 2002, 15(4 Pt 1):333-339.

33. Saha C, Eckert GJ, Ambrosius WT, et al.: Improvement in blood pressure with inhibition of the epithelial sodium channel in blacks with hypertension. Hypertension 2005, 46(3):481-487.

34. Sharabi Y, Adler E, Shamis A, et al.: Efficacy of add-on aldosterone receptor blocker in uncontrolled hypertension. Am J Hypertens 2006, 19(7):750-755.

35. Reisin E, Weir MR, Falkner B, et al.: Lisinopril versus hydrochlorothiazide in obese hypertensive patients: a multicenter placebo-controlled trial. Treatment in Obese Patients With
Hypertension (TROPHY) Study Group. Hypertension 1997, 30 (1 Pt 1):140-145.

36. Grassi G, Seravalle G, Dell'Oro R, et al.: Comparative effects of candesartan and hydrochlorothiazide on blood pressure, insulin sensitivity, and sympathetic drive in obese hypertensive individuals: results of the CROSS study. J Hypertens 2003, 21 (9):1761-1769.

37. Jordan J, Engeli S, Boschmann M, et al.: Hemodynamic and metabolic responses to valsartan and atenolol in obese hypertensive patients. J Hypertens 2005, 23(12):2313-2318.

38. Jordan J, Engeli S, Boye SW, et al.: Direct renin inhibition with aliskiren in obese patients with arterial hypertension. Hypertension 2007, 49(5):1047-1055.

39. Elliott WJ, Meyer PM: Incident diabetes in clinical trials of antihypertensive drugs: a network meta-analysis. Lancet 2007, 369(9557):201-207.

40. Cooper-DeHoff RM, Wen S, Beitelshees AL, et al.: Impact of abdominal obesity on incidence of adverse metabolic effects associated with antihypertensive medications. Hypertension, 55 (1):61-68

41. Neter JE, Stam BE, Kok FJ, et al.: Influence of weight reduction on blood pressure: a meta-analysis of randomized controlled trials. Hypertension 2003, 42(5):878-884.

42. •- Pimenta E, Gaddam KK, Oparil S, et al.: Effects of dietary sodium reduction on blood pressure in subjects with resistant hypertension: results from a randomized trial. Hypertension 2009, 54(3):475-481. This small but elegant study shows that excessive sodium intake can importantly contribute to resistance to antihypertensive treatment. The authors conclude that strategies that substantially reduce dietary salt intake should be part of the overall treatment of resistant hypertension.

43. Aucott L, Poobalan A, Smith WC, et al.: Effects of weight loss in overweight/obese individuals and long-term hypertension outcomes: a systematic review. Hypertension 2005, 45(6): $1035-1041$.

44. Haslam DW, James WP: Obesity. Lancet 2005, 366(9492):11971209.

45. Scholze J, Grimm E, Herrmann D, et al.: Optimal treatment of obesity-related hypertension: the Hypertension-Obesity-Sibutramine (HOS) study. Circulation 2007, 115(15):1991-1998.

46. Biaggioni I: Should we target the sympathetic nervous system in the treatment of obesity-associated hypertension? Hypertension 2008, 51(2):168-171.

47. Mason JM, Dickinson HO, Nicolson DJ, et al.: The diabetogenic potential of thiazide-type diuretic and beta-blocker combinations in patients with hypertension. J Hypertens 2005, 23(10):17771781.

48. ALLHAT Officers and Coordinators for the ALLHAT Collaborative Research Group. The Antihypertensive and Lipid-Lowering Treatment to Prevent Heart Attack Trial: Major outcomes in high-risk hypertensive patients randomized to angiotensin-converting enzyme inhibitor or calcium channel blocker vs diuretic: The Antihypertensive and Lipid-Lowering Treatment to Prevent Heart Attack Trial (ALLHAT). JAMA 2002, 288(23):2981-2997.

49. Stump CS, Hamilton MT, Sowers JR: Effect of antihypertensive agents on the development of type 2 diabetes mellitus. Mayo Clin Proc 2006, 81(6):796-806. 